\title{
Retesting for severe acute respiratory coronavirus virus 2 (SARS-CoV-2): Patterns of testing from a large US healthcare system
}

\author{
Amy J. Kennedy MD, MS², Mary K. Hilmes MBA², Linda Waddell RN, MSN², Alexandrea B. Bartow RN, MSN, ACNP-BC, \\ $M_{B A}^{3}$, Carla M. Baxter RN, BSN², Christiane M. Hadi MD, MPH, MSc ${ }^{4}$, Graham M. Snyder MD, MS ${ }^{4}$ and \\ Jessica S. Merlin MD, MBA, PhD ${ }^{1}$ \\ ${ }^{1}$ Division of General Internal Medicine, University of Pittsburgh School of Medicine, Pittsburgh, Pennsylvania, ${ }^{2}$ Wolff Center at the University of Pittsburgh \\ Medical Center, Pittsburgh, Pennsylvania, ${ }^{3}$ Department of Cardiothoracic Surgery, University of Pittsburgh Medical Center, Pittsburgh, Pennsylvania and \\ ${ }^{4}$ Division of Infectious Diseases, University of Pittsburgh School of Medicine, Pittsburgh, Pennsylvania
}

To the Editor - Coronavirus disease 2019 (COVID-19), a respiratory illness caused by severe acute respiratory syndrome coronavirus 2 (SARS-CoV-2), has caused a global pandemic, leading to significant morbidity and mortality. ${ }^{1,2}$ Accurate testing is essential to the identification and treatment of new cases of COVID-19 in the inpatient and outpatient settings.

In the United States, the initial focus of COVID-19 testing has been on ensuring adequate access to large-scale testing via a public health approach. However, given the limitations in efforts to ensure widespread access, individual hospitals and healthcare systems have worked to ensure that enough tests are available to meet clinical demand. Often decisions on who to test are left to individual clinicians, which leads to questions about when and who to retest for COVID-19, how often false positives or negatives might occur, and the duration of positivity. ${ }^{3}$

Research regarding why retesting for SARS-COV-2 might be indicated or what results might be expected is lacking. This report describes patterns of SARS-CoV-2 nucleic acid polymerase chain reaction (PCR) retesting in inpatients and outpatients within a large US healthcare system. We aimed to learn more about potential reasons for retesting and test characteristics.

\section{Methods}

We performed a retrospective chart review of all inpatients and outpatients aged $\geq 18$ years receiving care within the University of Pittsburgh Medical Center (UPMC) with $\geq 2$ SARS-CoV-2 PCR tests with an initial test between March 3 and May 3, 2020, and a subsequent test before May 21, 2020. UPMC operates 40 academic, community, and specialty hospitals and 700 doctors' offices and outpatient sites across Pennsylvania, New York, and Maryland. Widespread testing within UPMC at individual clinician discretion became available in March 2020, and recommended asymptomatic screening of preoperative patients began in May 2020.

We collected demographic characteristics, setting of care, reason for retesting, certain COVID-19 risk factors (ie, nursing home resident, immunocompromised, healthcare worker, COVID-19 exposure, travel history), and the date of tests, allowing for calculation of time between tests. PCR testing was performed using a lab-derived assay and through a commercial laboratory.

Author for correspondence: Jessica S. Merlin, E-mail: merlinjs@upmc.edu.

Cite this article: Kennedy AJ, et al. (2021). Retesting for severe acute respiratory coronavirus virus 2 (SARS-CoV-2): Patterns of testing from a large US healthcare system. Infection Control \& Hospital Epidemiology, 42: 1023-1025, https://doi.org/10.1017/ ice. 2020.413
Descriptive statistics were performed overall and for 4 groups: (1) initial positive test, any subsequent result(s) positive; (2) initial positive test, any subsequent result(s) negative; (3) initial negative test, any subsequent result(s) negative; and (4) initial negative test, any subsequent result(s) positive. These groups were not mutually exclusive and were constructed to learn as much as possible about testing characteristics. For example, within group 1, the potential length of time a test could remain positive (even if a subsequent test was then negative). The University of Pittsburgh Institutional Review Board approved this study.

\section{Results}

Among $>30,000$ initial tests, 485 were repeated; 259 were inpatients $(53.6 \%)$ and 230 were outpatients $(46.7 \%)$ at the time of initial test. Most individuals (348, 71.9\%) had 2 tests and $136(28 \%)$ had $\geq 3$ tests. Most patients were white (78\%), aged $41-80$ years (71.6\%), and had symptoms of fever (35.1\%), cough (37.2\%), or shortness of breath $(32.0 \%)$ at baseline (Table 1$)$.

Among 74 patients with an initial positive test, 35 (47\%) had any subsequent positive result (group 1) and 39 (53\%) had any subsequent negative result (group 2). The median time between an initial and last positive test was 18 days (interquartile range [IQR], 13; range, 2-39), and the median time between an initial positive and first negative test was 23 days (IQR, 12; range, 3-43). The most common reason for repeat testing was inpatient discharge planning, followed by discontinuation of inpatient isolation (Table 1).

Among 418 patients with an initial negative test, only $15(3.6 \%)$ had any subsequent positive result (group 4), while 403 (96.4\%) had any subsequent negative result (group 3). The most common reason for repeat testing was preoperative asymptomatic screening $(\mathrm{N}=154,31.3 \%)$, followed by clinical suspicion for a false negative $(\mathrm{N}=108,22.0 \%)$. For those who went from negative to positive, median time between tests was 8 days (IQR, 12; range, 1-23).

\section{Discussion}

In this retrospective study of a large US healthcare system, we found that retesting for SARS-CoV-2 was uncommon and often resulted in multiple negative tests. Most individuals were retested due to preprocedural asymptomatic screening or clinical suspicion for COVID-19 disease. In this population, PCR positivity persisted for a median of 18 to 23 days, and repeat testing after an initial negative test infrequently yielded a positive result. Prior studies have suggested that PCR positivity may persist beyond symptoms or infectivity; our findings suggest a potential time frame for this persistence. ${ }^{4}$ Most repeat

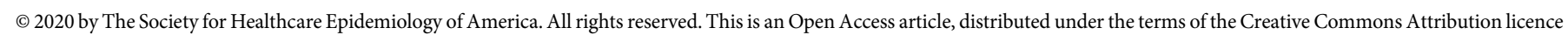
(http://creativecommons.org/licenses/by/4.0/), which permits unrestricted re-use, distribution, and reproduction in any medium, provided the original work is properly cited. 
Table 1. Sample Characteristics of 485 Participants With Repeat SARS-CoV-2 Testing

\begin{tabular}{|c|c|c|c|c|c|}
\hline Characteristic & $\begin{array}{c}\text { Total } \\
(\mathrm{N}=492)^{\mathrm{a}}\end{array}$ & $\begin{array}{l}\text { Group 1: Initial Positive, } \\
\text { Any Subsequent Positive } \\
(\mathrm{N}=35) \text {, No. }(\%)\end{array}$ & $\begin{array}{l}\text { Group 2: Initial Positive, } \\
\text { Any Subsequent Negative } \\
(\mathrm{N}=39), \text { No. }(\%)\end{array}$ & $\begin{array}{l}\text { Group 3: Initial Negative, } \\
\text { Any Subsequent Negative } \\
(\mathrm{N}=403), \text { No. }(\%)\end{array}$ & $\begin{array}{l}\text { Group 4: Initial Negative, } \\
\text { Any Subsequent Positive } \\
\qquad(\mathrm{N}=15), \text { No. }(\%)\end{array}$ \\
\hline Sex, male & $208(42.3)$ & $21(60.0)$ & $22(56.4)$ & $157(39.0)$ & $8(53.3)$ \\
\hline Age, median y & 60.5 & 63 & 58 & 56 & 65 \\
\hline $0-20$ & $6(1.2)$ & $1(2.9)$ & $1(2.6)$ & $4(1.0)$ & $0(0.0)$ \\
\hline $21-40$ & $100(20.3)$ & $6(17.1)$ & $8(20.5)$ & $83(20.6)$ & $3(20.0)$ \\
\hline $61-80$ & $176(35.8)$ & $18(51.4)$ & $18(46.2)$ & $133(33.0)$ & $7(46.7)$ \\
\hline$\geq 81$ & $34(6.9)$ & $3(8.6)$ & $1(2.6)$ & $28(6.9)$ & $2(13.3)$ \\
\hline \multicolumn{6}{|c|}{ Location at time of initial test } \\
\hline Outpatient & $259(52.6)$ & $22(62.9)$ & $20(51.3)$ & $210(52.1)$ & $7(46.7)$ \\
\hline Healthcare worker & $63(12.8)$ & $3(8.6)$ & $2(5.1)$ & $56(13.9)$ & $2(13.3)$ \\
\hline $\begin{array}{l}\text { Nursing home } \\
\text { resident }\end{array}$ & $44(8.9)$ & $5(14.3)$ & $8(20.5)$ & $28(6.9)$ & $3(20.0)$ \\
\hline Travel history & $14(2.8)$ & $6(17.1)$ & $4(10.3)$ & $4(1.0)$ & $0(0.0)$ \\
\hline Immunocompromised & $36(7.3)$ & $2(5.7)$ & $4(10.3)$ & $30(7.4)$ & $0(0.0)$ \\
\hline $\begin{array}{l}\text { Days between tests, } \\
\text { median (IQR) }\end{array}$ & $13(2-18)$ & $18(10.5-23.5)$ & $23(17-29)$ & $4(3-15)$ & $8(2-14)$ \\
\hline \multicolumn{6}{|l|}{ Reason for retesting } \\
\hline Clinical suspicion & $108(22.0)$ & $2(5.7)$ & $6(15.4)$ & $96(23.8)$ & $4(26.7)$ \\
\hline $\begin{array}{l}\text { Asymptomatic } \\
\text { screen }\end{array}$ & $154(31.3)$ & $0(0.0)$ & $0(0.0)$ & $153(38.0)$ & $1(6.7)$ \\
\hline
\end{tabular}

aSome patients fell into $>1$ result group, hence the total of $492>485 ; 492=$ number of entries analyzed; $485=$ number of unique entries.

tests ordered after an initial negative test were also negative, which is consistent with other emerging findings. ${ }^{5,6}$

The main limitation of this study is that testing was conducted only in individuals in whom it was clinically indicated, and only at the clinician's discretion, which limited our ability to draw conclusions about differences between test groups or to calculate a true false-negative rate.

In summary, we found that retesting for SARS-CoV-2 was rare and usually resulted in multiple negative tests. Future research should work to identify predictors of initial false negatives and to provide a more refined estimation of duration of infectivity.

Acknowledgments. We thank Ethan Lennox, MA, Division of General Internal Medicine, University of Pittsburgh School of Medicine, for his help with manuscript editing, and Amy Lukanski, DNP, RN, CPN, Bonnie Anton, RN, MN, Anna Marie Scekeres, RN, MSN, Tracy L. Morris, RN, BSN, Deborah Pesanka, RN, MN, and Audrey Paul, RN, MSN, Wolff Center at the University of Pittsburgh Medical Center, Pittsburgh, Pennsylvania, for their contributions to data extraction for this study.

Financial support. The first author was supported by a HRSA T32 training grant (grant no. T32HP22240) for the duration of the data analysis and writing of the manuscript.

Conflicts of interest. The authors declare no conflict of interest.

\section{References}

1. Arentz M, Yim E, Klaff L, et al. Characteristics and outcomes of 21 critically ill patients with COVID-19 in Washington state. JAMA 2020;323:1612-1614.

2. Garg S, Kim L, Whitaker M, et al. Hospitalization rates and characteristics of patients hospitalized with laboratory-confirmed coronavirus disease 2019Covid-net, 14 states, March 1-30, 2020. Morb Mortal Wkly Rep 2020;69:458-464.

3. Esbin MN, Whitney ON, Chong S, Maurer A, Darzacq X, Tjian R. Overcoming the bottleneck to widespread testing: a rapid review of nucleic 
acid testing approaches for COVID-19 detection. RNA 2020. doi: 10.1261/ rna.076232.120.

4. Lan L, Xu D, Ye G, et al. Positive RT-PCR test results in patients recovered from COVID-19. JAMA 2020;323:1502-1503.

5. Kucirka LM, Lauer SA, Laeyendecker O, Boon D, Lessler J. Variation in false-negative rate of reverse transcriptase polymerase chain reaction-based
SARS-CoV-2 tests by time since exposure. Ann Intern Med 2020. doi: 10.7326/ m20-1495.

6. Doll ME, Pryor R, MacKey D, et al. Utility of retesting for diagnosis of SARS-CoV-2/COVID-19 in hospitalized patients: impact of the interval between tests. Infect Control Hosp Epidemiol 2020;41:859-861.

\title{
Lack of nosocomial transmission to exposed inpatients and coworkers in an investigation of five SARS-CoV-2-infected healthcare workers
}

\author{
Kwadwo Mponponsuo $\mathrm{MD}^{1}$ (1), Geneviève Kerkerian $\mathrm{MD}^{1}$, Ranjani Somayaji MD, MPH ${ }^{1,2,5}$, Bayan Missaghi MD, MSc ${ }^{1,7}$, \\ Joseph V. Vayalumkal MD ${ }^{6,7}$, Oscar E. Larios MD ${ }^{1,7,8}$, Byron M. Berenger MD, MSc ${ }^{8,9}$, Matt Lauzon MD ${ }^{1,10}$, \\ Nicole McDonnell BNSc, RN ${ }^{10}$ and John Conly MD, $\mathrm{PhD}^{1,2,3,4,5,7,8}$ \\ ${ }^{1}$ Department of Medicine, Cumming School of Medicine, University of Calgary and Alberta Health Services, Calgary, Alberta, Canada, ${ }^{2}$ Department of \\ Microbiology, Immunology and Infectious Diseases, University of Calgary, Calgary, Alberta, Canada, ${ }^{3}$ Department of Community Health Sciences, University of \\ Calgary, Calgary, AB, Canada, ${ }^{4} \mathrm{O}$ 'Brien Institute for Public Health, University of Calgary and Alberta Health Services, Calgary, AB, Canada, ${ }^{5}$ Snyder Institute for \\ Chronic Diseases, University of Calgary and Alberta Health Services, Calgary, AB, Canada, ${ }^{6}$ Department of Pediatrics, Cumming School of Medicine, University \\ of Calgary and Alberta Health Services, Calgary, Alberta, Canada, ${ }^{7}$ Infection Prevention and Control, Alberta Health Services, Calgary, Alberta, Canada, \\ ${ }^{8}$ Department of Pathology and Laboratory Medicine, Cumming School of Medicine, University of Calgary and Alberta Health Services, Calgary, Alberta, Canada, \\ ${ }^{9}$ Alberta Public Health Laboratory, Alberta Precision Laboratories, Calgary, Alberta, Canada and ${ }^{10}$ Workplace Health and Safety, Alberta Health Services, Calgary, \\ Alberta, Canada
}

To the Editor-Clinical equipoise remains regarding the optimal protective measures and equipment to prevent nosocomial transmission risk of severe acute respiratory coronavirus virus 2 (SARS-CoV-2), the causative agent of coronavirus disease 2019 (COVID-19). ${ }^{1}$ Underpinning this question is debate regarding airborne versus droplet transmission of the virus. We evaluated 5 consenting healthcare workers (HCWs) who were diagnosed with community-acquired COVID-19 and who had interacted with patients and other HCWs $(n=72)$ while symptomatic or presymptomatic in the Calgary Health Zone of Alberta Health Services between March 1 and April 15, 2020.

Approval from the University of Calgary Ethics Committee (no. REB20-0510) was obtained to conduct interviews following verbal consent using a standardized case report form and questionnaire. Index HCWs and their patient and coworker exposures (Supplementary Table S1 online) were identified through databases and tracing with the infection prevention and control and occupational health departments. We utilized a risk assessment adapted from previously published guidance for contact tracing. We deemed close contact an interaction of $>15$ minutes at a distance of $<1 \mathrm{~m}^{2}$ Those exposed to the index HCWs were followed for 30 days for compatible SARS-CoV-2 infection symptom

Author for correspondence: John Conly, E-mail: john.conly@albertahealthservices.ca or jconly@ucalgary.ca

Cite this article: Mponponsuo K, et al. (2021). Lack of nosocomial transmission to exposed inpatients and coworkers in an investigation of five SARS-CoV-2-infected healthcare workers. Infection Control \& Hospital Epidemiology, 42: 1025-1026, https:// doi.org/10.1017/ice.2020.392 development. SARS-CoV-2 test results were obtained on exposed individuals who developed symptoms. Testing for SARS-CoV-2 was performed using a multiplex reverse-transcriptase real-time polymerase chain reaction (RT-PCR) targeting the envelope and the RNA-dependent RNA polymerase encoding regions ( $\mathrm{E}$ and RdRp genes). ${ }^{3}$

All 5 of the HCWs (ie, HCWs A-E) had tested positive for SARS-CoV-2 by RT-PCR with E gene cycle threshold (Ct) values between 10.9 and 30.2 via nasopharyngeal or deep nasal swab and had symptoms prior to or on the day they worked (Supplementary Table S2 online). HCWs B and E worked 2 days while symptomatic, and the remainder worked 1 day. HCW A developed symptoms of mild nasal and sinus congestion the day of her shift; HCW $\mathrm{B}$ developed a sore throat 5 days prior to the day he worked; HCW $\mathrm{C}$ had a fever and cough develop while at work; HCW D developed fever, chills, and rhinorrhea the evening following her shift; and HCW E had sneezing, headache, fatigue, and sore throat on the days she worked.

Between the index cases, a total of 39 HCWs (Supplementary Table S1 online) were exposed (range, 6-12 per HCW). All index cases interacted with at least 5 other HCWs at a distance of $<1 \mathrm{~m}$ for $>15$ minutes. Of the exposed HCWs who underwent testing $(\mathrm{n}=16)$, none tested positive for SARS-CoV-2 in the follow-up period. Notably, HCW B was undergoing training and partnered with another HCW for 2 hours, providing direct patient care.

In total, 33 patients were exposed to the index cases (range, 2-24) (Supplementary Table S1 online). HCW E did not have any patient exposure. Of the patients exposed to HCW A, 20 of $24(83 \%)$ were deemed close contacts. All of the patients of HCWs B, C, and D were exposed for $>15$ minutes at $<1 \mathrm{~m}$ distance for the described interactions. Only HCW C and D wore a mask for all of their patient interactions $(n=6)$. Of 22 patients who

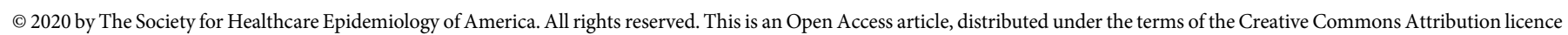
(http://creativecommons.org/licenses/by/4.0/), which permits unrestricted re-use, distribution, and reproduction in any medium, provided the original work is properly cited. 